\title{
ON SOME EQUATIONS RELATED TO DERIVATIONS IN RINGS
}

\author{
JOSO VUKMAN AND IRENA KOSI-ULBL
}

Received 30 December 2004 and in revised form 21 July 2005

Let $m$ and $n$ be positive integers with $m+n \neq 0$, and let $R$ be an $(m+n+2)$ !-torsion free semiprime ring with identity element. Suppose there exists an additive mapping $D$ : $R \rightarrow R$, such that $D\left(x^{m+n+1}\right)=(m+n+1) x^{m} D(x) x^{n}$ is fulfilled for all $x \in R$, then $D$ is a derivation which maps $R$ into its center.

Throughout this paper, $R$ will represent an associative ring with center $Z(R)$. A ring $R$ is $n$-torsion free, where $n>1$ is an integer, in case $n x=0, x \in R$ implies $x=0$. As usual the commutator $x y-y x$ will be denoted by $[x, y]$. We will use basic commutator identities $[x y, z]=[x, z] y+x[y, z]$ and $[x, y z]=[x, y] z+y[x, z]$. Recall that a ring $R$ is prime if $a R b=(0)$ implies that either $a=0$ or $b=0$, and is semiprime if $a R a=(0)$ implies $a=0$. An additive mapping $D: R \rightarrow R$ is called a derivation if $D(x y)=D(x) y+x D(y)$ for all pairs $x, y \in R$, and is called a Jordan derivation in case $D\left(x^{2}\right)=D(x) x+x D(x)$ for all $x \in R$. Every derivation is a Jordan derivation. The converse is in general not true. A classical result of Herstein [11, Theorem 3.1] asserts that any Jordan derivation on a 2-torsion free prime ring is a derivation (see [7] for an alternative proof). Cusack [9, Corollary 5] has generalized Herstein's theorem to 2-torsion free semiprime rings (see [4] for an alternative proof). A mapping $F$ of a ring $R$ into itself is called commuting (centralizing) on $R$ in case $[F(x), x]=0([F(x), x] \in Z(R))$ holds for all $x \in R$. The theory of commuting and centralizing mappings was initiated by a result of Posner [12, Theorem 2] (Posner's second theorem), which states that the existence of a nonzero centralizing derivation $D: R \rightarrow R$, where $R$ is a prime ring, forces the ring to be commutative.

Vukman has proved the following result.

Theorem 1 [13, Theorem 3]. Let $R$ be a 2-and 3-torsion free noncommutative prime ring with identity element, and let $D: R \rightarrow R$ be an additive mapping such that $D\left(x^{3}\right)=3 x D(x) x$ holds for all $x \in R$. In this case $D=0$.

Let us point out that any commuting derivation on an arbitrary ring satisfies the relation $D\left(x^{3}\right)=3 x D(x) x$. Theorem 1 was the motivation for the result. 
Theorem 2. For integers $m$, $n$ with $m \geq 0, n \geq 0$, and $m+n \neq 0$, let $R$ be an $(m+n+2)$ !torsion free semiprime ring with identity element. Suppose there exists an additive mapping $D: R \rightarrow R$, such that $D\left(x^{m+n+1}\right)=(m+n+1) x^{m} D(x) x^{n}$ is fulfilled for all $x \in R$. In this case, $D$ is a derivation, which maps $R$ into its center. In case $R$ is a noncommutative prime ring, we have $D=0$.

In case $m=1, n=0$ (we adopt the convention $x^{0}=e$, for all $x \in R$, where $e$ denotes the identity element), we have an additive mapping satisfying the relation $D\left(x^{2}\right)=2 x D(x)$, $x \in R$. Such mappings are called left Jordan derivations (see $[8,10,15])$. Brešar and Vukman [8, Corollary1.3] have proved that the existence of a nonzero Jordan derivation on a 2- and 3-torsion free prime ring forces the ring to be commutative. For the proof of Theorem 2, we need Theorem 4, which is of independent interest. For the proof of Theorem 4 the lemma below will be needed. We refer the reader to [3] for the definitions and an account of the theory of the extended centroid and central closure as well as related topics and to [6] for an introductory survey on functional identities.

Lemma 3. Let $R$ be a 2-torsion free prime ring and let $A$ be its central closure. Suppose that an additive mapping $F: R \rightarrow A$ satisfies $[[F(x), x], x]=0$ for all $x \in R$. Then, $[F(x), x]=0$ holds for all $x \in R$.

Proof. In the case when $F$ maps into $R$, the lemma was first proved by Brešar in [5, Theorem 2]. Fortunately, the same proof works in the case when $F$ maps into $A$ (on the other hand, see, e.g., [2] for a more general result).

Theorem 4. Let $R$ be a 2-torsion free semiprime ring. Suppose that an additive mapping $F: R \rightarrow R$ satisfies $[[F(x), x], x]=0$ for all $x \in R$. Then, $[F(x), x]=0$ holds for all $x \in R$.

Proof. Since $R$ is semiprime, there exists a family of prime ideals $\left\{P_{\alpha} ; \alpha \in A\right\}$ such that $\cap_{\alpha} P_{\alpha}=(0)$. Moreover, without loss of generality, we may assume that the prime rings $R_{\alpha}=R / P_{\alpha}$ are 2-torsion free (see, e.g., [1, page 459]). Now fix some $P=P_{\alpha}, \alpha \in A$. The theorem will be proved by showing that $[F(x), x] \in P$ for every $x \in R$. Given $x \in R$, we will write $\bar{x}$ for the coset $x+P \in R / P$. By $C$, we denote the extended centroid of the prime ring $R / P$, and by $A$ the central closure of $R / P$. One can consider $A$ as a vector space over the field $C$. Since $C$ can be regarded as a subspace of $A$, there exists a subspace $B$ of $A$ such that $A=B+C$. We denote by $\pi$ the canonical projection of $A$ onto $B$. Substituting $x+p$ for $x$ in $[[F(x), x], x]=0$, it follows at once that $[[F(p), x], x] \in P$ for all $x \in R, p \in P$, that is, $[[\overline{F(p)}, \bar{x}], \bar{x}]=0$. Using Posner's theorem [12, Theorem 2] (or just [5, Lemma 2] for that matter), it follows that $[\overline{F(p)}, \bar{x}]=0$ for all $x \in R, p \in P$, that is, $\overline{F(p)}$ lies in the center of $R / P$. In particular, $\pi \overline{F(p)}=0$. Using this, we see that the mapping $\bar{F}: R / P \rightarrow A$, $\bar{F}(\bar{x})=\pi \overline{F(x)}$ is well defined. Note that $\bar{F}$ is additive and satisfies $[[\bar{F}(\bar{x}), \bar{x}], \bar{x}]=0$ for all $x \in R$. But then the lemma shows that $[\bar{F}(\bar{x}), \bar{x}]=0$ for all $x \in R$, which implies that $[F(x), x] \in P$. The proof of the theorem is complete.

Theorem 4 generalizes Theorem 2 proved by Brešar [5] and Theorem 2 proved by Vukman in [14].

Proof of Theorem 2. From the relation

$$
D\left(x^{m+n+1}\right)=(m+n+1) x^{m} D(x) x^{n}, \quad x \in R,
$$


it follows immediately that

$$
D(e)=0
$$

where $e$ denotes the identity element. Putting $x+e$ for $x$ in the relation (1) and using (2), we obtain

$$
\begin{aligned}
& \sum_{i=0}^{m+n+1}\left(\begin{array}{c}
m+n+1 \\
i
\end{array}\right) D\left(x^{m+n+1-i}\right) \\
& \quad=(m+n+1)\left(\sum_{i=0}^{m}\left(\begin{array}{c}
m \\
i
\end{array}\right) x^{m-i}\right) D(x)\left(\sum_{i=0}^{n}\left(\begin{array}{c}
n \\
i
\end{array}\right) x^{n-i}\right), \quad x \in R .
\end{aligned}
$$

Using (1) and collecting together terms of (3) involving the same number of factors of $e$, we obtain

$$
\sum_{i=1}^{m+n} f_{i}(x, e)=0, \quad x \in R
$$

where $f_{i}(x, e)$ stands for the expression of terms involving $i$ factors of $e$.

Replacing $x$ by $x+2 e, x+3 e, \ldots, x+(m+n) e$ in turn in (1) and expressing the resulting system of $m+n$ homogeneous equations, we see that the coefficient matrix of the system is a van der Monde matrix

$$
\left[\begin{array}{cccc}
1 & 1 & \cdots & 1 \\
2 & 2^{2} & \cdots & 2^{m+n} \\
\vdots & \vdots & \vdots & \vdots \\
m+n & (m+n)^{2} & \cdots & (m+n)^{m+n}
\end{array}\right] .
$$

Since the determinant of the matrix is different from zero, it follows that the system has only a trivial solution.

In particular,

$$
\begin{aligned}
f_{m+n-1}(x, e)= & \left(\begin{array}{l}
m+n+1 \\
m+n-1
\end{array}\right) D\left(x^{2}\right) \\
- & (m+n+1)\left(\left(\begin{array}{c}
m \\
m-1
\end{array}\right)\left(\begin{array}{l}
n \\
n
\end{array}\right) x D(x)+\left(\begin{array}{c}
m \\
m
\end{array}\right)\left(\begin{array}{c}
n \\
n-1
\end{array}\right) D(x) x\right)=0, \quad x \in R, \\
f_{m+n-2}(x, e)= & \left(\begin{array}{l}
m+n+1 \\
m+n-2
\end{array}\right) D\left(x^{3}\right) \\
& -(m+n+1)\left(\left(\begin{array}{c}
m \\
m-2
\end{array}\right)\left(\begin{array}{l}
n \\
n
\end{array}\right) x^{2} D(x)+\left(\begin{array}{c}
m \\
m-1
\end{array}\right)\left(\begin{array}{c}
n \\
n-1
\end{array}\right) x D(x) x\right. \\
& \left.+\left(\begin{array}{c}
m \\
m
\end{array}\right)\left(\begin{array}{c}
n \\
n-2
\end{array}\right) D(x) x^{2}\right)=0, \quad x \in R .
\end{aligned}
$$


2706 On some equations related to derivations in rings

Since $R$ is a $(m+n+2)$ !-torsion free ring, the above equations reduce to

$$
\begin{array}{rl}
(m+n) D\left(x^{2}\right)=2 m x & D(x)+2 n D(x) x, \quad x \in R, \\
(m+n)(m+n-1) D\left(x^{3}\right)= & 3 m(m-1) x^{2} D(x)+6 m n x D(x) x \\
& +3 n(n-1) D(x) x^{2}, \quad x \in R,
\end{array}
$$

respectively. We intend to prove that the mapping $x \mapsto[D(x), x]$ is commuting on $R$. For this purpose, we write in $x+y$ for $x$ in (8), which gives

$$
(m+n) D(x y+y x)=2 m x D(y)+2 m y D(x)+2 n D(x) y+2 n D(y) x, \quad x, y \in R .
$$

Putting $y=(m+n) x^{2}$ in the relation above, we obtain

$$
\begin{aligned}
(m+n)^{2} D\left(x^{3}\right)= & m(m+n) x D\left(x^{2}\right)+m(m+n) x^{2} D(x) \\
& +n(m+n) D(x) x^{2}+n(m+n) D\left(x^{2}\right) x, \quad x \in R .
\end{aligned}
$$

According to (8), the above relation reduces to

$$
(m+n)^{2} D\left(x^{3}\right)=\left(3 m^{2}+m n\right) x^{2} D(x)+4 m n x D(x) x+\left(3 n^{2}+m n\right) D(x) x^{2}, \quad x \in R .
$$

Subtracting (9) from (12), we obtain

$$
(m+n) D\left(x^{3}\right)=m(n+3) x^{2} D(x)-2 m n x D(x) x+n(m+3) D(x) x^{2}, \quad x \in R .
$$

From the above relation, we obtain

$$
\begin{aligned}
(m+n)^{2} D\left(x^{3}\right)= & (m+n) m(n+3) x^{2} D(x)-2(m+n) m n x D(x) x \\
& +(m+n) n(m+3) D(x) x^{2}, \quad x \in R .
\end{aligned}
$$

Subtracting (14) from (12), one obtains

$m n(m+n+2) x^{2} D(x)-2 m n(m+n+2) x D(x) x+m n(m+n+2) D(x) x^{2}=0, \quad x \in R$

Since $R$ is $(m+n+2)$ !-torsion free ring, the above relation reduces to

$$
D(x) x^{2}+x^{2} D(x)-2 x D(x) x=0, \quad x \in R,
$$

which can be written in the form

$$
[[D(x), x], x]=0, \quad x \in R
$$

Now Theorem 4 makes it possible to conclude that

$$
[D(x), x]=0, \quad x \in R
$$


In other words, $D$ is commuting on $R$. The fact that $D$ is commuting on $R$ makes it possible to replace $D(x) x$ in (8) by $x D(x)$. The relation (8) reduces to $D\left(x^{2}\right)=2 x D(x)$, $x \in R$. Using again the fact that $D$ is commuting, we obtain $D\left(x^{2}\right)=D(x) x+x D(x), x \in$ $R$. In other words, $D$ is a Jordan derivation. Let us recall that any Jordan derivation on a 2-torsion free semiprime ring is a derivation. It is well known and easy to prove that any commuting derivation on a semiprime ring $R$ maps $R$ into $Z(R)$ (see [15]). In case $R$ is a noncommutative prime ring, Posner's second theorem completes the proof of the theorem.

In the proof of Theorem 2, we met an additive mapping $D$ satisfying the relation below

$$
(m+n) D\left(x^{2}\right)=2 m D(x) x+2 n x D(x) .
$$

In case $n=0$ and $R$ is an $m$-torsion free ring, we have an additive mapping $D$ satisfying the relation $D\left(x^{2}\right)=2 x D(x), x \in R$. In other words, $D$ is a left Jordan derivation. It was proved (see [15, Theorem 1]) that left Jordan derivations on a 2- and 3-torsion free semiprime ring are derivations which map the ring into its center. These observations lead to the conjecture.

Conjecture 5. Let $R$ be a semiprime ring with suitable torsion restrictions. Suppose there exists an additive mapping $D: R \rightarrow R$ satisfying the relation

$$
(m+n) D\left(x^{2}\right)=2 n D(x) x+2 m x D(x),
$$

for all $x \in R$ and some integers $m \geq 0, n \geq 0, m+n \neq 0$. In case $m \neq n$, the mapping $D$ is a derivation which maps $R$ into $Z(R)$.

Our next result is related to the conjecture above.

Theorem 6. Let $R$ be a $2, m, n, m+n$, and $|m-n|$-torsion free semiprime ring, and let $D: R \rightarrow R$ be an additive mapping satisfying the relation

$$
(m+n) D(x y)=2 m D(x) y+2 n x D(y)
$$

for all pairs $x, y \in R$ and some integers $m \geq 0, n \geq 0, m+n \neq 0$. In case $m \neq n$, we have $D=0$.

Proof. We have the relation

$$
(m+n) D(x y)=2 m D(x) y+2 n x D(y), \quad x, y \in R .
$$

We compute the expression $(m+n)^{2} D(x y x)$ in two ways. First we obtain (using (22))

$$
\begin{aligned}
(m+n)^{2} D(x(y x)) & =2 m(m+n) D(x) y x+2 n(m+n) x D(y x) \\
& =2 m(m+n) D(x) y x+2 n x(2 m D(y) x+2 n y D(x)), \quad x, y \in R .
\end{aligned}
$$

Thus we have

$$
(m+n)^{2} D(x y x)=2 m(m+n) D(x) y x+4 m n x D(y) x+4 n^{2} x y D(x), \quad x, y \in R .
$$


2708 On some equations related to derivations in rings

On the other hand, we have (using (22))

$$
\begin{aligned}
(m+n)^{2} D((x y) x) & =2 m(m+n) D(x y) x+2 n(m+n) x y D(x) \\
& =2 m(2 m D(x) y+2 n x D(y)) x+2 n(m+n) x y D(x), \quad x, y \in R .
\end{aligned}
$$

Thus we have

$$
(m+n)^{2} D(x y x)=4 m^{2} D(x) y x+4 m n x D(y) x+2 n(m+n) x y D(x), \quad x, y \in R .
$$

Subtracting the relation (24) from the relation (26), we obtain

$$
m(m-n) D(x) y x+n(m-n) x y D(x)=0, \quad x, y \in R,
$$

which reduces to

$$
m D(x) y x+n x y D(x)=0, \quad x, y \in R
$$

Putting $y x$ for $y$ in the relation (28), we obtain

$$
m D(x) y x^{2}+n x y x D(x)=0, \quad x, y \in R
$$

Right multiplication of the relation (28) by $x$ gives

$$
m D(x) y x^{2}+n x y D(x) x=0, \quad x, y \in R
$$

Subtracting the relation (29) from the relation (30), we obtain

$$
n(x y(D(x) x-x D(x)))=0, \quad x, y \in R,
$$

which gives

$$
x y[D(x), x]=0, \quad x, y \in R
$$

Writing in the relation (32) $D(x) y$ for $y$, then multiplying the relation (32) by $D(x)$ from the left-hand side and comparing the relations so obtained, we obtain

$$
[D(x), x] y[D(x), x]=0, \quad x, y \in R
$$

whence it follows

$$
[D(x), x]=0, \quad x \in R
$$

by semiprimeness of $R$. Putting $y=x$ in the relation (22) and using the relation (34), 
we obtain $D\left(x^{2}\right)=2 D(x) x, x \in R$, which can be written in the form

$$
D\left(x^{2}\right)=D(x) x+x D(x), \quad x \in R,
$$

because of (34). In other words, $D$ is a Jordan derivation. As we have already mentioned, any Jordan derivation on a 2-torsion free semiprime ring is a derivation. Now one can replace $D(x y)$ with $D(x) y+x D(y)$ in the left-hand side of (22), which gives

$$
D(x) y=x D(y), \quad x, y \in R
$$

Substituting $z x$ for $x$ in (36) gives

$$
D(z) x y=0, \quad x, y, z \in R,
$$

whence it follows first $D(z) x D(z)=0$ for all $x, z \in R$, and then by semiprimeness $D=0$. The proof of the theorem is complete.

\section{Acknowledgments}

The authors would like to thank Professor Matej Brešar for helpful information on the subject of this paper. This research has been supported by the Research Council of Slovenia.

\section{References}

[1] W. E. Baxter and W. S. Martindale, III, Jordan homomorphisms of semiprime rings, J. Algebra 56 (1979), no. 2, 457-471.

[2] K. I. Beidar, Y. Fong, P.-H. Lee, and T.-L. Wong, On additive maps of prime rings satisfying the Engel condition, Comm. Algebra 25 (1997), no. 12, 3889-3902.

[3] K. I. Beidar, W. S. Martindale, III, and A. V. Mikhalev, Rings with Generalized Identities, Monographs and Textbooks in Pure and Applied Mathematics, vol. 196, Marcel Dekker, New York, 1996.

[4] M. Brešar, Jordan derivations on semiprime rings, Proc. Amer. Math. Soc. 104 (1988), no. 4, 1003-1006.

[5] - On a generalization of the notion of centralizing mappings, Proc. Amer. Math. Soc. 114 (1992), no. 3, 641-649.

[6] _ Functional identities: a survey, Algebra and Its applications (Athens, Ohio, 1999), Contemp. Math., vol. 259, American Mathematical Society, Rhode Island, 2000, pp. 93-109.

[7] M. Brešar and J. Vukman, Jordan derivations on prime rings, Bull. Austral. Math. Soc. 37 (1988), no. 3, 321-322.

[8] - On left derivations and related mappings, Proc. Amer. Math. Soc. 110 (1990), no. 1, $7-16$.

[9] J. M. Cusack, Jordan derivations on rings, Proc. Amer. Math. Soc. 53 (1975), no. 2, 321-324.

[10] Q. Deng, On Jordan left derivations, Math. J. Okayama Univ. 34 (1992), 145-147 (1994).

[11] I. N. Herstein, Jordan derivations of prime rings, Proc. Amer. Math. Soc. 8 (1957), 1104-1110.

[12] E. C. Posner, Derivations in prime rings, Proc. Amer. Math. Soc. 8 (1957), 1093-1100.

[13] J. Vukman, Commuting and centralizing mappings in prime rings, Proc. Amer. Math. Soc. 109 (1990), no. 1, 47-52.

[14] Derivations on semiprime rings, Bull. Austral. Math. Soc. 53 (1996), no. 3, 353-359. 
2710 On some equations related to derivations in rings

[15]_, On some additive mappings in semiprime rings and Banach algebras, Aequationes Math. 58 (1999), 1-10.

Joso Vukman: Department of Mathematics, Faculty of Education (PEF), University of Maribor, Koroška 160, 2000 Maribor, Slovenia

E-mail address: joso.vukman@uni-mb.si

Irena Kosi-Ulbl: Department of Mathematics, Faculty of Education (PEF), University of Maribor, Koroška 160, 2000 Maribor, Slovenia

E-mail address: irena.kosi@uni-mb.si 


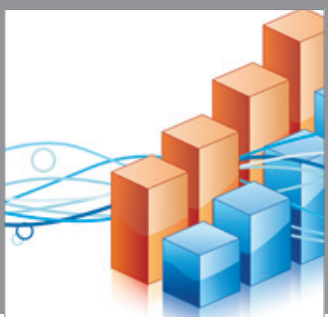

Advances in

Operations Research

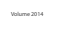

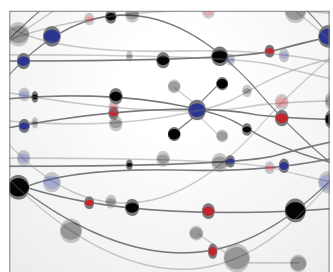

\section{The Scientific} World Journal
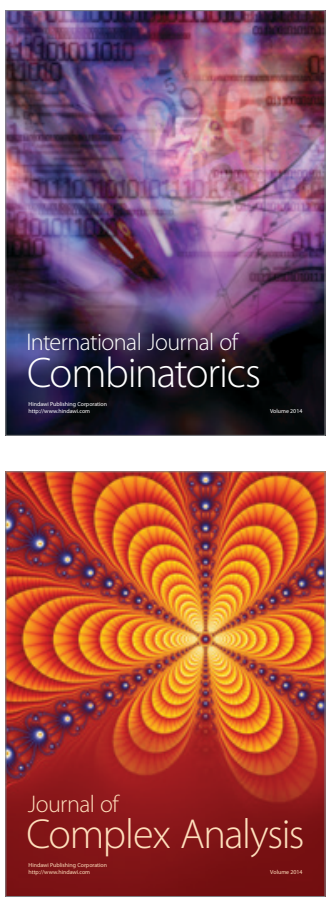

International Journal of

Mathematics and

Mathematical

Sciences
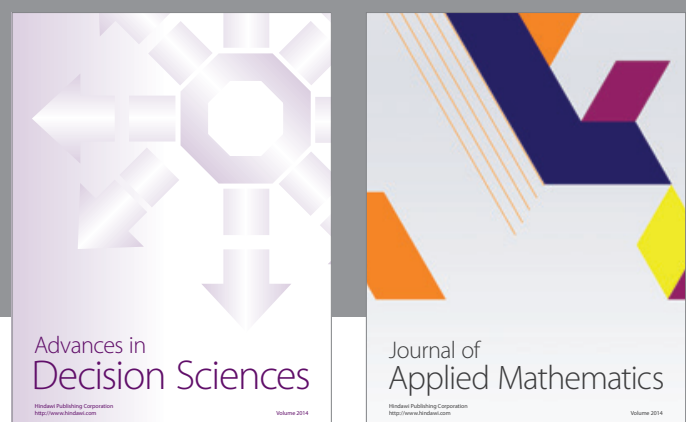

Journal of

Applied Mathematics
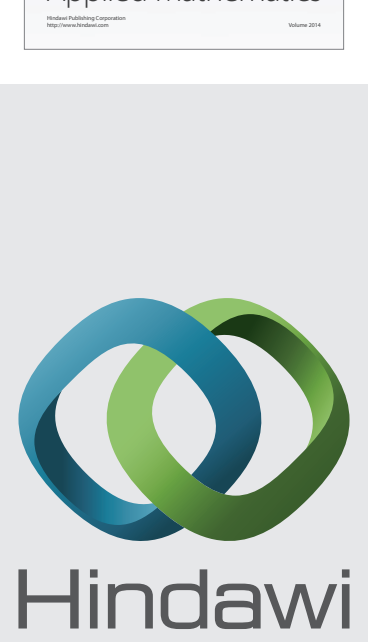

Submit your manuscripts at http://www.hindawi.com
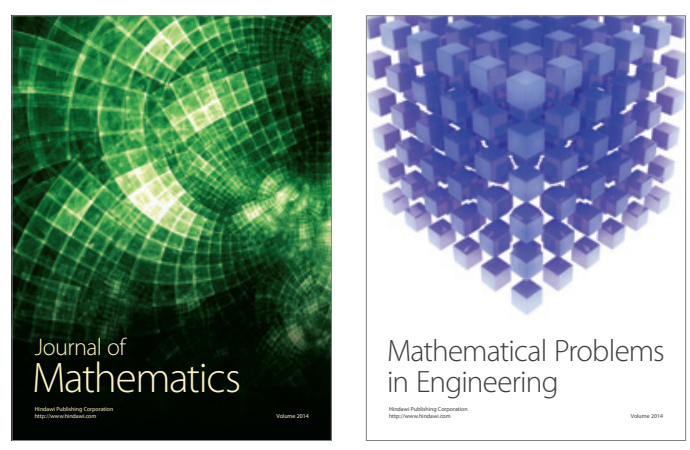

Mathematical Problems in Engineering
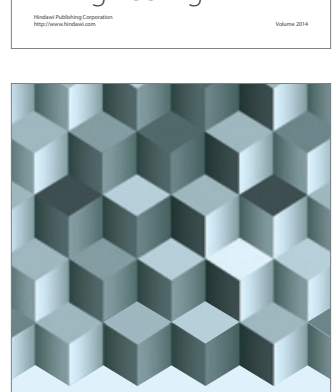

Journal of

Function Spaces
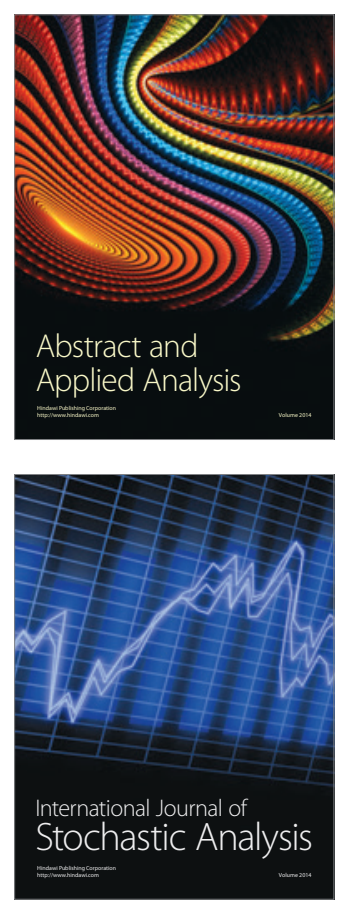

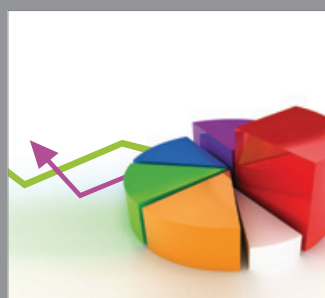

ournal of

Probability and Statistics

Promensencen
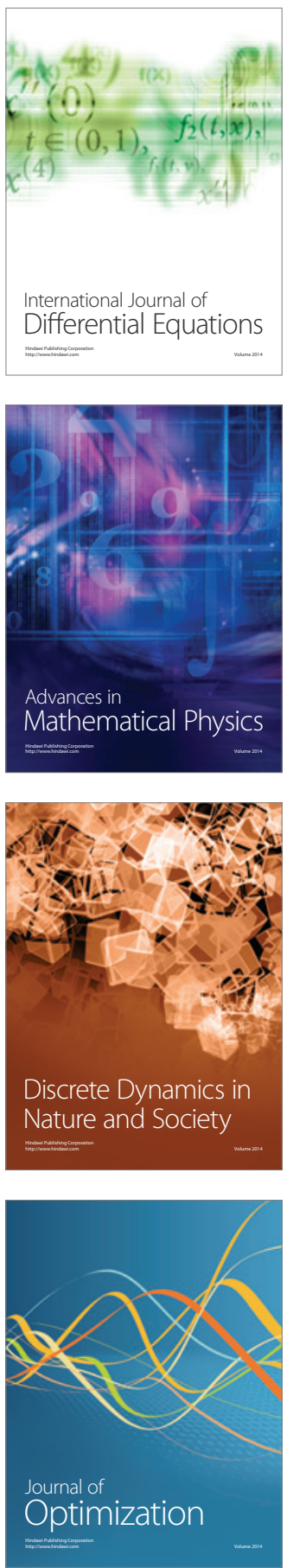